\title{
Analysis of Stock Splits Based on Risk Theory: Empirical Evidence from the Chinese Stock Markets
}

\author{
Shujin WU \\ MOE Key Laboratory of Advanced Theory and Application in Statistics and Data Science, School of \\ Statistics, East China Normal University, Shanghai 200062, China \\ E-mail: sjwu@stat.ecnu.edu.cn
}

Tong XU

School of Statistics, East China Normal University, Shanghai 200062, China

E-mail: xu-tong@outlook.com

\begin{abstract}
The paper first analyzes price change due to stock splits in Chinese stock markets, which shows stock prices typically go up for stock splits. Then theoretical analyses based on risk theory are presented to explain the reason, where the method comes from a new perspective and obtained theoretical conclusions show that stock splits typically make stock price go up if risk-compensation function is convex, and go down if risk-compensation function is concave. Stock prices typically go up for stock splits because risk-compensation functions are mainly convex. The obtained conclusions are consistent with the known results in the last three decades.
\end{abstract}

Keywords stock split; risk theory; price; Chinese stock markets

\section{Introduction}

A stock split is a corporate action in which a company divides its existing shares into multiple shares to boost the liquidity of the shares. Although the number of shares outstanding increases by a specific multiple, the total dollar value of the shares remains the same compared to pre-split amounts, because the split does not add any real value.

Although the intrinsic value of stock is not changed by a stock split, the stock price typically goes up according to many known empirical analyses, so large numbers of investors happily accumulate large holdings of the stock this way, and some companies regularly split their stocks. The so-called intrinsic value is the perceived or calculated value of a company, including tangible and intangible factors, using fundamental analysis. Since stock split does not change the intrinsic value of stock, why does a stock price typically go up for a stock split? The answers mainly focus on that stock split boosts its liquidity, that is, stock split seems to make shares more affordable to small investors. However, increasing liquidity seems to be believable for stocks with high price, but unbelievable for stocks with lower price.

Received November 12, 2020, accepted January 25, 2021

Supported by the National Natural Science Foundation of China (11471120) and the Science and Technology Commission of Shanghai Municipality (19JC1420100) 
There is a strong contradiction between earlier and later empirical findings about whether a stock split can bring abnormal yield. In fact, Fama, Fisher and Jensen, et al. found no abnormal performance subsequent to stock splits ${ }^{[1]}$. However, both Ikenberry, Rankine and Stice, et al. reported abnormal returns of seven to eight percent in the 12 months following stock splits ${ }^{[2,3]}$. Byun and Rozeff measured the postsplit performance of 12747 stock splits from 1927 to 1996 using two methods to measure abnormal yields: Size and book-to-market reference portfolios with bootstrapping, and calendar-time abnormal yields combined with factor models, and they found these samples showed small or negligible abnormal yields using the calendartime method ${ }^{[4]}$. That is, stock split evidence against market efficiency was neither pervasive nor compelling.

In the last two decade, there are lots of literatures on stock split, many of which study the impact of stock splits on abnormal return and increasing liquidity. Li, Liu and Shi studied whether stock split may improve liquidity and found that institutional investors, especially short-term investors, increased their holding of the splitting firm's stock ${ }^{[5]}$. More literatures on stock split can refer to [6-16] etc. and their references.

Additionally, Schultz found strong evidence that trading costs increased, and weak evidence that costs of market making declined following stock splits ${ }^{[17]}$. Nguyen, Tran and Zeckhauser examined the 718 stock split events in the emerging stock market of Vietnam from 2007 through $2011^{[18]}$. They found evidence consistent with illegal insider trading, particularly in firms that were vulnerable to insider manipulation and, therefore, more likely to split their stocks. Chen, Nguyen and Singal found that stock splits in aggregate were followed by positive abnormal future earnings growth, suggesting that stock splits contained information about future, rather than past, operating performance ${ }^{[11]}$. Minnick and Raman investigated the reason that stock splits was declining ${ }^{[19]}$. Crutchley and Swidler investigated multiple reverse stock splits, and warned investors should beware multiple reverse stock splits ${ }^{[20]}$.

Up to now, we have not found either result that analyzes stock splits in the Chinese stock markets or method that analyzes stock splits based on risk theory. In the paper, we investigate 7700 stock returns with stock split and 25618 stock returns without stock split in the Chinese stock markets from 1999 to 2018. Our empirical analysis shows that stock prices typically go up for stock splits, which is consistent with the known empirical analysis results in the last three decades, such as [2], [3] and [4] etc., but contradicts to the earlier empirical findings in [1]. Then theoretical analyses based on risk theory are presented to explain the reason that stock splits typically bring abnormal returns. Our analysis method adopts a new perspective and is different from the known methods, which mainly contribute abnormal returns of stock splits to increasing liquidity or illegal insider trading. Our obtained conclusions show that stock splits typically make stock price go up if risk-compensation function is convex, and go down if risk-compensation function is concave. According to risk theory, risk-compensation functions are mainly convex, so stock prices typically go up for stock splits. Our obtained theoretical conclusions are consistent with the known empirical analysis in the last three decades. 


\section{Empirical Analysis on Stock Splits in Chinese Stock Markets}

In the section, we will present empirical analysis on the effect of stock splits in the Chinese stock markets, herein and hereafter the Chinese stock markets refers specifically to Shanghai Stock Exchange and Shenzhen Stock Exchange, excluding the Hong Kong Stock Exchange, etc., where the Shanghai Stock Exchange officially opened on Dec 19, 1990 and the Shenzhen Stock Exchange was put into trial operation on Dec 01, 1990.

\subsection{Sample Description}

Considering the Chinese stock markets were emerging markets in the first few years of 1990s and the Asian financial crisis broke out in 1998, we analyze impact effect of stock splits mainly based on stock data in the Chinese stock markets from 1999 to 2018, including 7700 stock returns with stock split and 25618 stock returns without stock split.

We will use the dividend distribution data of every stocks, and the daily yield data of every stocks, where the dividend distribution data of stocks are downloaded from the Cathay Taian Database from Jan 01, 1999 to Dec 31, 2018, and the daily yield data of every stocks are downloaded from the Wind Database from Jan 01, 1999 to Dec 31, 2018.

According to complex calculation using the dividend distribution data of every stocks and the daily yield data of every stocks, we can obtain the key variables as follows:

- Num1: The yearly number of stocks with stock split

- Yield1: The yearly average return of stocks with stock split

- Var1: The variance of yearly return of stocks with stock split

- Num2: The yearly number of stocks without stock split

- Yield2: The yearly average return of stocks without stock split

- Var2: The variance of yearly return of stocks without stock split

- diffYield: The difference of Yield1 and Yield2, i.e., diffYield $=$ Yield1 - Yield2

- Num: The total number of stocks, i.e., Num = Num1 + Num2

- Percent: The proportion of the number of stocks with stock split to the total number of all stocks, i.e., Percent $=\frac{\text { Num1 }}{\text { Num }} \times 100 \%$

The detailed collated sample data refer to Table $1^{1}$.

\subsection{Analysis on the Number of Stocks with Stock Split}

Minnick and Raman pointed out the proportion of US companies undertaking stock splits drops sharply from a peak of about $23 \%$ in 1982 to less than $1 \%$ in $2009^{[19]}$. So we want to know what's about the proportion of companies undertaking stock splits in the Chinese stock markets.

\footnotetext{
${ }^{1}$ Table 1 was collated by the second author, Mr. Tong $\mathrm{Xu}$, using the software Python 3.7.
} 
Table 1 The collated sample data

\begin{tabular}{ccccccccc}
\hline Year & Num1 & Yield1 & Var1 & Num2 & Yield2 & Var2 & diffYield & Percent \\
\hline 1999 & 238 & $36.49 \%$ & 0.4894 & 686 & $15.44 \%$ & 0.3542 & $21.06 \%$ & $25.76 \%$ \\
2000 & 179 & $85.46 \%$ & 0.6667 & 882 & $63.03 \%$ & 0.4392 & $22.42 \%$ & $16.87 \%$ \\
2001 & 197 & $-14.01 \%$ & 0.2246 & 943 & $-23.63 \%$ & 0.1537 & $9.62 \%$ & $17.28 \%$ \\
2002 & 164 & $-11.62 \%$ & 0.2395 & 1043 & $-20.84 \%$ & 0.1602 & $9.22 \%$ & $13.59 \%$ \\
2003 & 150 & $2.31 \%$ & 0.3290 & 1117 & $-14.13 \%$ & 0.2766 & $16.44 \%$ & $11.84 \%$ \\
2004 & 239 & $-0.17 \%$ & 0.3141 & 1124 & $-18.35 \%$ & 0.2210 & $18.18 \%$ & $17.53 \%$ \\
2005 & 181 & $2.90 \%$ & 0.3098 & 1185 & $-15.38 \%$ & 0.2572 & $18.28 \%$ & $13.25 \%$ \\
2006 & 392 & $115.31 \%$ & 1.0559 & 1026 & $85.79 \%$ & 0.8456 & $29.52 \%$ & $27.64 \%$ \\
2007 & 279 & $241.86 \%$ & 1.8695 & 1238 & $193.95 \%$ & 1.2036 & $47.91 \%$ & $18.39 \%$ \\
2008 & 424 & $-53.23 \%$ & 0.3136 & 1153 & $-58.98 \%$ & 0.2323 & $5.75 \%$ & $26.89 \%$ \\
2009 & 248 & $151.07 \%$ & 0.8995 & 1432 & $145.02 \%$ & 0.9981 & $6.05 \%$ & $14.76 \%$ \\
2010 & 392 & $27.72 \%$ & 0.5261 & 1628 & $10.96 \%$ & 0.4697 & $16.76 \%$ & $19.41 \%$ \\
2011 & 626 & $-29.31 \%$ & 0.2355 & 1675 & $-29.80 \%$ & 0.2501 & $0.49 \%$ & $27.21 \%$ \\
2012 & 580 & $7.19 \%$ & 0.4865 & 1876 & $2.90 \%$ & 0.3940 & $4.29 \%$ & $23.62 \%$ \\
2013 & 467 & $51.75 \%$ & 0.7624 & 2003 & $22.55 \%$ & 0.4813 & $29.20 \%$ & $18.91 \%$ \\
2014 & 493 & $44.96 \%$ & 0.5569 & 2099 & $46.14 \%$ & 0.4432 & $-1.18 \%$ & $19.02 \%$ \\
2015 & 741 & $108.56 \%$ & 1.0180 & 2070 & $67.83 \%$ & 0.7887 & $40.73 \%$ & $26.36 \%$ \\
2016 & 595 & $-6.43 \%$ & 0.3425 & 2438 & $-10.23 \%$ & 0.3153 & $3.80 \%$ & $19.62 \%$ \\
2017 & 492 & $-11.09 \%$ & 0.3543 & 2973 & $-13.95 \%$ & 0.3369 & $2.86 \%$ & $14.20 \%$ \\
2018 & 623 & $-28.06 \%$ & 0.2556 & 2947 & $-32.28 \%$ & 0.2005 & $4.23 \%$ & $17.45 \%$ \\
\hline & & & & & & & &
\end{tabular}

Using Table 1, we draw the line charts of the number of stocks with stock split Num1 and the total number of stocks Num in Figure 1 and the line chart of the proportion of stocks with split to total number of stocks in the Chinese stock markets Percent in Figure 2, where Num $=$ Num1 + Num2.

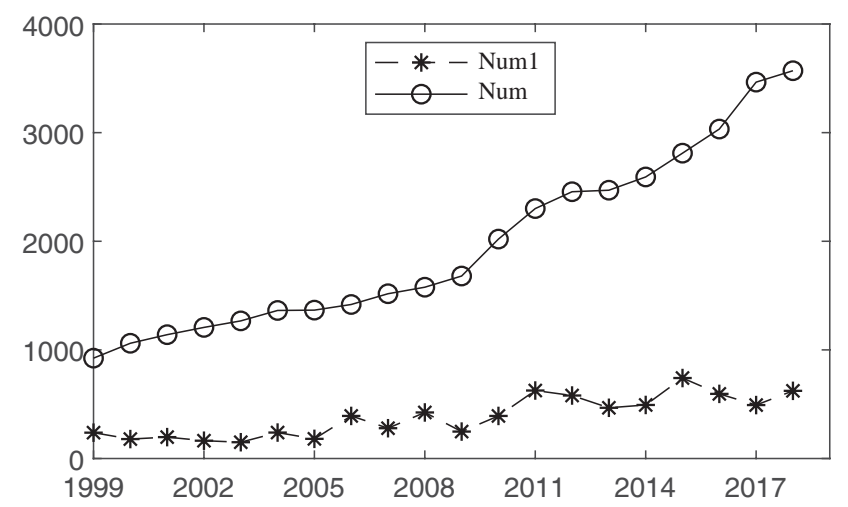

Figure 1 The line charts of Num1 and Num 


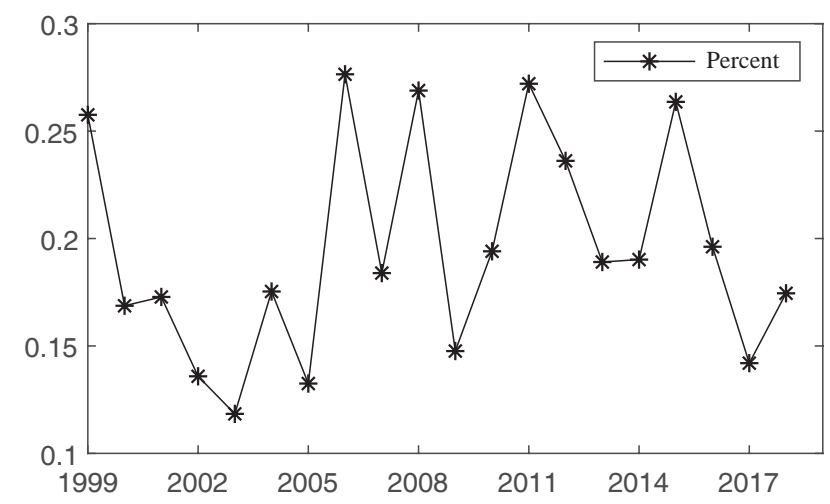

Figure 2 The line chart of Percent

Figure 1 and Figure 2 show the total number of stocks Num grows very rapidly and the proportion Percent keep stable intuitively.

Further, we will check the stationarity of Percent with Augmented Dickey-Fuller test (adftest) and Phillips-Perron test (pptest), herein and hereafter the italic word in bracket is the corresponding function of the software Matlab R2018b. For example, adftest is the function of Augmented Dickey-Fuller test in Matlab R2018b.

The null hypothesis and alternative hypothesis follows as

$$
H_{0} \text { : Percent has unit roots vs } \quad H_{1} \text { : Percent has no unit roots }
$$

Both Augmented Dickey-Fuller test and Phillips-Perron test obtain the same statistic value $=-1.0436$, the same critical value $=-1.9531$ and the same $p$-Value $=0.25783>0.05$, so we reject the null hypothesis $H_{0}$ and accept the alternative hypothesis $H_{1}$, which means Percent has no unit roots. That is, we accept Percent is stationary.

From the above analysis, we know the proportion of the number of stocks with stock split to the total number of all stocks is stationary in the Chinese stock markets, then the number of stocks with stock split Num1 is enough large. Thus, it makes sense that we analyse the impact effect of stock split.

\subsection{Analysis on the Yearly Average Returns}

In the subsection, we want to check whether the yearly average return of stocks with stock split Yield1 is really greater than the yearly average return of stocks without split Yield2.

Using Table 1, we draw the line charts of the yearly average return of stocks with stock split Yield1 and the yearly average return of stocks without split Yield2 in Figure 3.

Figure 3 shows that Yield1 is greater than Yield2 in all years except for 2014. That is, from the visual point of view, the yearly average return of stocks with stock split is almost greater than the yearly average return of stocks without stock split. We further check whether the yearly average return of stocks with stock split Yield1 is really greater than the yearly average return of stocks without split Yield2 by hypothesis test. 


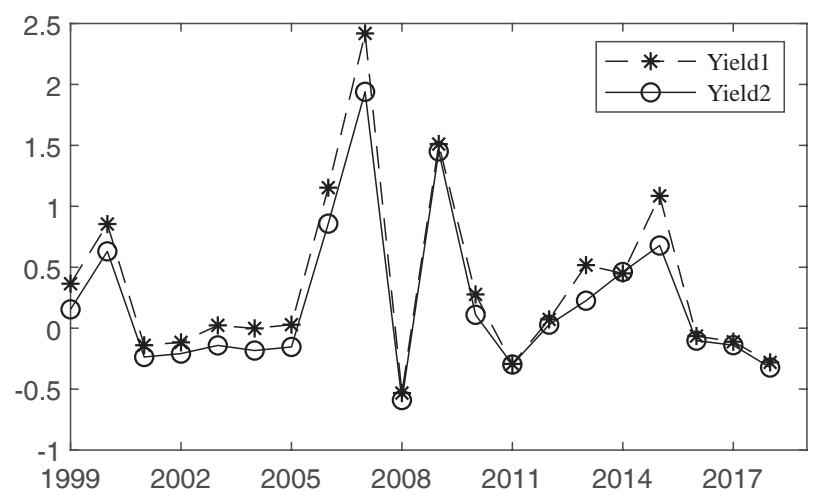

Figure 3 The line chart of Yield1 and Yield2

\subsubsection{Stationarity, Randomness and Normality Tests of Yield1 and Yield2}

We use Augmented Dickey-Fuller test (adftest) and Phillips-Perron test (pptest) to check the stationarity, Ljung-Box Q-test (lbqtest) to check the randomness, and Kolmogorov-Smirnov test (kstest) and Jarque-Bera test (jbtest) to check the normality. The test design refers to Table 2 , and the detailed test data follow as Table 3 .

Table 2 Design of stationarity, randomness and normality tests of Yield1 and Yield2

\begin{tabular}{llll}
\hline Test type & Test method & Null hypothesis & Alternative hypothesis \\
\hline \multirow{2}{*}{ Stationarity } & Augmented Dickey-Fuller test & Unit root exists & Unit root doesn't exist \\
& Phillips-Perron test & Unit root exists & Unit root doesn't exist \\
\hline Randomness & Ljung-Box Q-test & It is purely random & It is not purely random \\
\hline \multirow{2}{*}{ Normality } & Kolmogorov-Smirnov test & $\begin{array}{l}\text { It follows a normal } \\
\text { distribution }\end{array}$ & $\begin{array}{l}\text { It does't follow any normal } \\
\text { distribution }\end{array}$ \\
& Jarque-Bera test & $\begin{array}{l}\text { It follows a normal } \\
\text { distribution }\end{array}$ & $\begin{array}{l}\text { It does't follow any normal } \\
\text { distribution }\end{array}$ \\
\hline
\end{tabular}

Table 2 and Table 3 show that, as for stationarity test of Yield1 and Yield2, we reject the null hypothesis and accept the alternative hypothesis according to both Augmented Dickey-Fuller test and Phillips-Perron test, that is, we accept both Yield1 and Yield2 are stationary; As for randomness test of Yield1 and Yield2, we accept the null hypothesis and reject the alternative hypothesis according to Ljung-Box Q-test, that is, we accept both Yield1 and Yield2 are purely random; As for normality test of Yield1 and Yield2, we accept the null hypothesis and reject the alternative hypothesis according to Kolmogorov-Smirnov test, that is, we accept both Yield1 and Yield2 follow some normal distributions. However, we reject the null hypothesis and accept the alternative hypothesis according to Jarque-Bera test, that is, we don't accept either Yield1 or Yield2 follows any normal distribution. Unfortunately, we have obtained two contradictory test conclusion about the normality of Yield1 and Yield2. 
Table 3 Stationarity, randomness and normality tests of Yield1 and Yield2

\begin{tabular}{lllllll}
\hline Variable & Test type & Function & $\begin{array}{l}\text { Statistic } \\
\text { value }\end{array}$ & $\begin{array}{l}\text { Critical } \\
\text { value }\end{array}$ & $p$-Value & $h$-Value \\
\hline \multirow{4}{*}{ Yield1 } & \multirow{2}{*}{ Stationarity } & adftest & -3.6235 & -1.9531 & 0.0011 & 1 \\
\cline { 2 - 7 } & Rptest & -3.6235 & -1.9531 & 0.0011 & 1 \\
\cline { 2 - 7 } & Randomness & lbqtest & 8.8080 & 30.1440 & 0.9765 & 0 \\
\hline \multirow{2}{*}{ Normality } & kstest & 7.5712 & 3.8011 & 0.0163 & 0 \\
& \multirow{2}{*}{ Stationarity } & jbtest & 0.2052 & 0.2941 & 0.3235 & 1 \\
\cline { 2 - 7 } & Randomness & lbqtest & -4.1502 & -1.9531 & 0.0010 & 1 \\
\cline { 2 - 7 } & \multirow{2}{*}{ Normality } & kstest & 7.3783 & 3.8011 & 0.0171 & 0 \\
\hline
\end{tabular}

\subsubsection{Homogeneity of Variance Test of Yield1 and Yield2}

Because we have obtained the contradictory conclusion about the normality of Yield1 and Yield2 with Kolmogorov-Smirnov test and Jarque-Bera test, we want to check whether the variance of Yield1, Var1, equals the variance of Yield2, Var2, under the conditions that both Yield1 and Yield2 have normality.

Using Table 1 we draw the line charts of Var1 and Var2 in Figure 4 and the line chart of Var1 in Figure 5.

Figure 4 and Figure 5 show that Var1 is greater than Var2 in all years except for 2009 and 2011. That is, the variance of Yield1 is almost greater than the variance of Yield2 intuitively. We further check whether the variance of Yield1 equals the variance of Yield2 by the two-sample $F$-test for equal variances (vartest2). The null hypothesis is that Yield1 and Yield2 come from normal distributions with the same variance and the alternative hypothesis is that Yield1 and Yield2 come from normal distributions with different variances. The function vartest2 obtains that test statistic $F=1.302335$, rejection domain $W=\{F<0.51548$ or $F>3.2903\}$ and $p$-Value $=0.57045$, so we accept the null hypothesis and reject the alternative hypothesis. That is, we accept that both Yield1 and Yield2 follow normal distributions with the same variance.

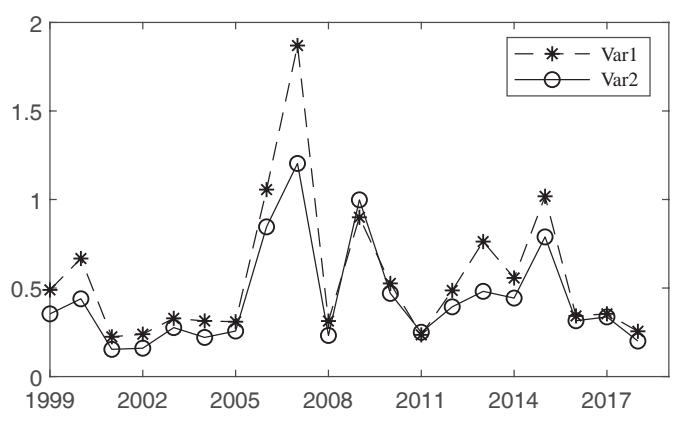

Figure 4 The line chart of Var1 and Var2 


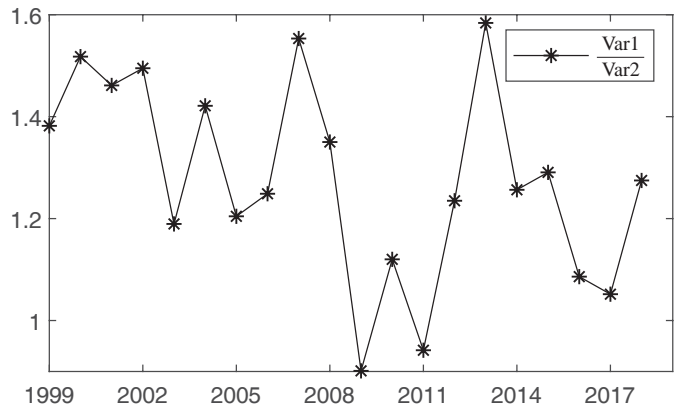

Figure 5 The line chart of $\frac{\operatorname{Var} 1}{\operatorname{Var} 2}$

\subsubsection{Test of Yield1 $>$ Yield2}

Under the condition that both Yield1 and Yield2 follow normal distributions with the same variance, we can further check whether Yield1 > Yield2 by two-sample $t$-test (ttest2). The null hypothesis is Yield1 $\leq$ Yield2 and the alternative hypothesis is Yield1 $>$ Yield2. The function ttest2(Yield1, Yield2, 'Alpha', 0.05, 'Tail', 'left') obtains the test result of statistic value $=0.71027$, rejection domain $W=(-\infty, 0.51556)$ and $p$-Value $=0.75906$, so we reject the null hypothesis and accept the alternative hypothesis, i.e., we accept Yield1 > Yield2.

In sum, we accept that stock splits can bring some abnormal yields in a statistical sense if we can accept both Yield1 and Yield2 follow normal distributions, though Kolmogorov-Smirnov test shows either Yield1 or Yield2 doesn't follow any normal distribution.

\subsection{Analysis on the Difference of Yearly Average Returns}

Although the previous subsection presents that the yearly average return of stocks with stock split Yield1 is greater than the yearly average return of stocks without stock split Yield2, it needs the conditions that both Yield1 and Yield2 have normality. However, Jarque-Bera test shows Yield1 or Yield2 doesn't follow any normal distribution though Kolmogorov-Smirnov test shows both Yield1 and Yield2 follows normal distributions. Thus, the results of the previous subsection are not so sure. In the subsection, we analyse whether diffYield is greater than zero, where diffYield $=$ Yield1 - Yield2, which is equivalent to whether Yield1 is greater than Yield2, but they need different conditions.

Using Table 1, we draw the line chart of the difference of diffYield in Figure 6.

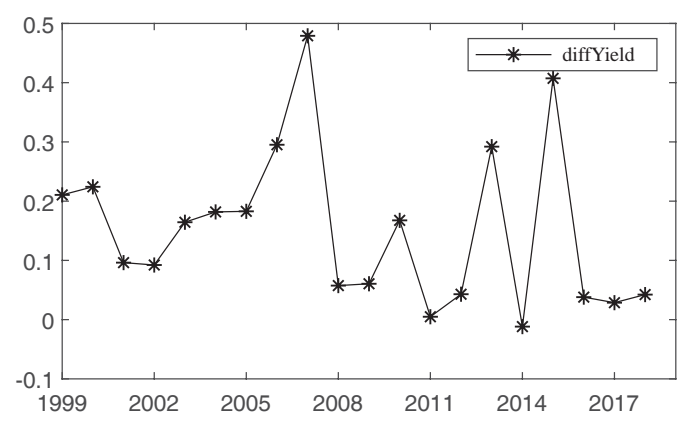

Figure 6 The line chart of diffYield 
Figure 6 shows that diffYield is greater than zero in all years except for 2014. That is, from the visual point of view, diffYield is almost greater than zero. In the following we will check whether diffYield is really greater than zero by hypothesis test.

\subsubsection{Stationarity, Randomness and Normality Tests of diffYield}

We also use Augmented Dickey-Fuller test and Phillips-Perron test to check the stationarity, Ljung-Box Q-test to check the randomness, and Kolmogorov-Smirnov test and Jarque-Bera test to check the normality. The test design refers to Table 2 , and the detailed test data follow as Table 4.

Table 4 Stationarity, randomness and normality tests of diffYield

\begin{tabular}{cccccc}
\hline Test Type & Test function & Statistic Value & Critical Value & $p$-Value & $h$-Value \\
\hline \multirow{2}{*}{ Stationarity } & adftest & -2.5033 & -1.9531 & 0.0152 & 1 \\
& pptest & -2.5033 & -1.9531 & 0.0152 & 1 \\
\hline \multirow{2}{*}{ Randomness } & lbqtest & 10.3520 & 30.1440 & 0.9437 & 0 \\
\hline \multirow{2}{*}{ Normality } & kstest & 0.1621 & 0.2941 & 0.6123 & 0 \\
& jbtest & 2.7386 & 3.8011 & 0.0808 & 0 \\
\hline
\end{tabular}

Table 4 shows that, as for stationarity test of diffYield, we reject the null hypothesis and accept the alternative hypothesis according to both Augmented Dickey-Fuller test and PhillipsPerron test, that is, we accept diffYield is stationary; As for randomness test of diffYield, we accept the null hypothesis and reject the alternative hypothesis according to Ljung-Box Q-test, that is, we accept diffYield is purely random; As for normality test of diffYield, we accept the null hypothesis and reject the alternative hypothesis according to both Kolmogorov-Smirnov test and Jarque-Bera test, that is, we accept diffYield follows a normal distribution.

In fact, the quantile-quantile plot for normal distribution of diffYield follows as Figure 7.

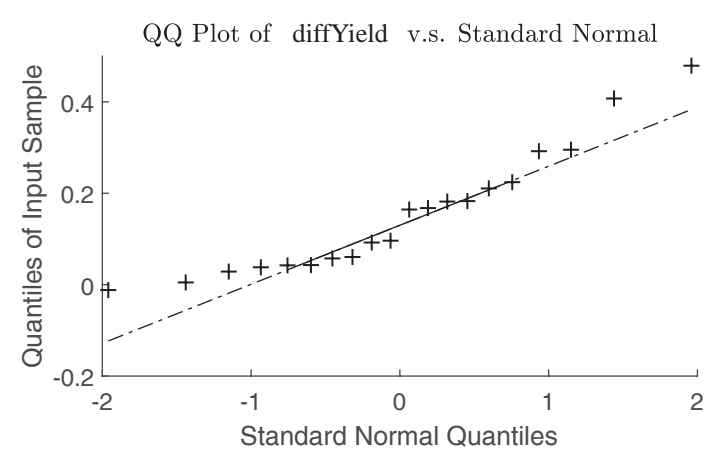

Figure 7 The quantile-quantile plot of diffYield

Figure 7 is almost a straight line, suggesting that diffYield follows a normal distribution. 


\subsubsection{Test of diffYield $>0$}

Since we have accepted that diffYield follows a normal distribution, we can further check whether diffYield $>0$ by one-sample $t$-test (ttest). The null hypothesis is diffYield $\leq 0$ and the alternative hypothesis is diffYield $>0$. The function ttest(diffYield, 'Alpha', 0.05, 'Tail', 'le $\left.f t^{\prime}\right)$ obtains the test result of statistic value $=5.0515$, rejection domain $W=(-\infty, 0.20513)$ and $p$-Value $=0.99996$, so we reject the null hypothesis and accept the alternative hypothesis, i.e., we accept diffYield $>0$.

In sum, we accept diffYield $>0$ in a statistical sense. That is, we accept that stock splits really bring some abnormal yields in a statistical sense.

\section{Theoretical Analysis on Stock Split According to Risk Theory}

In Section 2 we have pointed out stock splits can bring some abnormal yields by the empirical analysis of 7700 stock splits in the Chinese stock markets from 1999 to 2018, which is consistent with many known empirical analysis results, such as [2], [3] and [4], etc., but contradicts the earlier empirical findings in [1].

In the last two decades, there are lots of literatures researching stock splits and analyzing the reasons that stock splits can bring some abnormal yields, and mainly contribute abnormal yields of stock splits to increasing liquidity ${ }^{[5]}$ or illegal insider trading ${ }^{[18]}$. We know increasing liquidity may bring abnormal yields for high-price stocks but it has little effect on low-price stocks, and illegal insider trading occurs only in a few stock splits. Thus, it is not enough to explain abnormal yields of stock splits with increasing liquidity or illegal insider trading. In the paper, we will establish a reasonable price model to explain the reason that stock prices typically go up for stock splits in the view of risk theory, which is a new perspective.

In a risk-aversive market, denote the risk-compensation function of a rational investor by $\eta(z)$, where $z$ is the risk size, then $\eta(z)$ is increasing and satisfies $\eta(0)=0$. Assume a risky asset has intrinsic value $X$, where $X$ is a random variable defined in $(0,+\infty)$. And denote the risk size by $\rho(X)$, where $\rho$ is some risk measure. Obviously, $\rho(X) \geq 0$ for any $X$.

The so-called reasonable price for a risky asset just reflects the exchange value of the risky asset in financial markets, in other words, the reasonable price is the price that the rational investor is willing to pay after deducting risk compensation. We denote the reasonable price of risky asset with intrinsic value $X$ by $P_{r}(X)$. According to risk theory, for any rational investor the reasonable price of risky asset with intrinsic value $X$ follows as

$$
P_{r}(X)=E[X]-\eta(\rho(X)),
$$

where $E[\cdot]$ means taking the mathematical expectation. The reasonable price model (1) denotes that, for any rational investor, the reasonable price equals the value of the expectation of intrinsic value minus its risk compensation. Particularly, in a risk-neutral market, $\eta(\cdot) \equiv 0$ and

$$
P_{r}(X)=E[X]
$$

If $\eta(\rho(X)) \geq E[X]$, then $V(X) \leq 0$, which means that the risky asset has no investment value for risk-aversive investors. Thus, we always assume $0 \leq \eta(\rho(X))<E[X]$ in the paper.

It is very easy to obtain two properties from (1) as follows. 
Proposition 1 Assume the risk size remains unchanged, then the reasonable price will change the same amount as that of expectation value of intrinsic value.

In fact, we denote the intrinsic value just before its expectation value changing by $X_{0}$ and just after the its expectation value changing by $X_{1}$. If $\eta\left(\rho\left(X_{0}\right)\right)=\eta\left(\rho\left(X_{1}\right)\right)$, then it yields from (1) that

$$
P_{r}\left(X_{1}\right)-P_{r}\left(X_{0}\right)=E\left[X_{1}\right]-E\left[X_{0}\right] .
$$

That is, the reasonable price will change the same amount as that of expectation value of intrinsic value while the risk size remains unchanged.

Proposition 2 Assume the mathematical expectation of intrinsic value remains unchanged, then the reasonable price will change the opposite direction to that of risk size of intrinsic value.

In fact, we also denote the intrinsic value just before its risk size changing by $X_{0}$ and just after its risk size changing by $X_{1}$. If $E\left[X_{0}\right]=E\left[X_{1}\right]$, then it follows from (1) that

$$
P_{r}\left(X_{1}\right)-P_{r}\left(X_{0}\right)=-\left(\eta\left(\rho\left(X_{1}\right)\right)-\eta\left(\rho\left(X_{0}\right)\right)\right) \text {. }
$$

That is, the reasonable price will change the opposite direction to that of risk size of intrinsic value while the mathematical expectation of intrinsic value remains unchanged.

In the following, we will analyze how the stock price changes for a stock split according to the risk theory.

\subsection{Price Analysis for a Stock Split}

Assume a stock of intrinsic value $X$ will be split by the strategy that a share is split into $m$ shares, where $m>1$. Denote $\alpha=\frac{1}{m}$, then $\alpha \in(0,1)$. And denote the stock price and risk size just before the stock split by $P_{0}$ and $\rho_{0}$ respectively, then its ex-right price and risk size are $\alpha P_{0}$ and $\alpha \rho_{0}$.

In a risk-aversive financial market, for a rational investor we can regard the stock price just before the stock split equals its reasonable price. It yields from (1) that

$$
P_{0}+\eta\left(\rho_{0}\right)=E[X]
$$

If the risk-compensation function $\eta$ is convex, then it follows from (1), $\eta(0)=0$ and $\alpha \in(0,1)$ that

$$
\alpha P_{0}+\eta\left(\alpha \rho_{0}\right) \leq \alpha P_{0}+\alpha \eta\left(\rho_{0}\right)=\alpha E[X],
$$

which means stock price should go up for a stock split.

If the risk-compensation function $\eta$ is concave, then it follows from (1), $\eta(0)=0$ and $\alpha \in(0,1)$ that

$$
\alpha P_{0}+\eta\left(\alpha \rho_{0}\right) \geq \alpha P_{0}+\alpha \eta\left(\rho_{0}\right)=\alpha E[X],
$$

which means stock price should go down for a stock split.

According to the above analysis, we obtain the following conclusion.

Theorem 1 For a stock split, if the risk-compensation function is convex, then stock price should go up. If the risk-compensation function is concave, then stock price should go down.

According to risk theory, for a risk-aversive investor the risk-compensation function is mainly convex, so stock price typically goes up for a stock split by Theorem 1, which is a new perspective to explain the impact effect of stock splits. 


\subsection{Price Analysis for Stock Split and Expectation of Intrinsic Value Change}

If some information that affects its future intrinsic value of stock, such as expected return, unexpected calamity and inflation etc, is issued at the same time as the stock split information, then its expectation of intrinsic value will changes while stock split occurs. As for this case, we have the following conclusion.

Theorem 2 Assume risk-compensation function is $\eta$, the risk size and the increment of expectation of intrinsic value just before ex-right are $\rho_{0}$ and $\Delta$. Then

1) its price should go down for a stock split if and only if $\alpha \eta\left(\rho_{0}\right)-\eta\left(\alpha \rho_{0}\right)<\alpha \Delta$.

2) its price should go up for a stock split if and only if $\alpha \eta\left(\rho_{0}\right)-\eta\left(\alpha \rho_{0}\right)>\alpha \Delta$.

3) a stock split doesn't make its price changed if and only if $\alpha \eta\left(\rho_{0}\right)-\eta\left(\alpha \rho_{0}\right)=\alpha \Delta$.

The proof refers to Appendix A.1.

Theorem 2 shows when the expectation of future intrinsic value and stock split simultaneously occur the stock price changes very complicatedly. It could go up, go down or remain unchanged. The actual change depends on the quantitative relationship between the expectation of future intrinsic value and effect of stock split.

\subsection{Price Analysis for a Stock Split and Risk Size Change}

If some information that affects its risk size of stock, such as risky investment, upstream and downstream industry chain information, and industry risk etc, is issued at the same time as the stock split information, then its risk size will change while stock split occurs. As for this case, we have the following conclusion.

Theorem 3 Assume risk-compensation function is $\eta$, the risk size and the increment of risk size just before ex-right are $\rho_{0}$ and $\delta$. Then

1) its price should go down for a stock split if and only if $\eta\left(\alpha\left(\rho_{0}+\delta\right)\right)>\alpha \eta\left(\rho_{0}\right)$.

2) its price should go up for a stock split if and only if $\eta\left(\alpha\left(\rho_{0}+\delta\right)\right)<\alpha \eta\left(\rho_{0}\right)$.

3) a stock split doesn't make its price changed if and only if $\eta\left(\alpha\left(\rho_{0}+\delta\right)\right)=\alpha \eta\left(\rho_{0}\right)$.

The proof refers to Appendix A.2.

Theorem 3 shows when the risk size and stock split simultaneously occur the stock price changes very complicatedly, too. It maybe go up, go down or remain unchanged. The actual change depends on the quantitative relationship between the risk size and effect of stock split.

\section{Conclusion}

The paper mainly researches two problems: One is how stock splits impact on stock price in Chinese stock markets. The other is to explain the reason that stock splits impact their stock prices.

By comparing 7700 stock returns with stock splits and 25618 stock returns without stock splits from 1999 to 2018, we find that stock prices typically go up for stock splits in the Chinese stock markets. The obtained conclusion is consistent with the known empirical analysis results 
in the last three decades, such as [2], [3] and [4], etc., but contradicts to the earlier empirical findings in [1].

As for the reason that stock splits impact their stock prices, we analyze it with risk theory. Our analysis method adopts a new perspective and is different from the known methods, which mainly contribute abnormal returns of stock splits to increasing liquidity or illegal insider trading. Our obtained conclusions show that stock splits typically make stock price go up if risk-compensation function is convex, and go down if risk-compensation function is concave. According to risk theory, risk-compensation functions are mainly convex, so stock prices typically go up for stock splits. Our obtained theoretical conclusions are consistent with the known empirical analysis in the last three decades.

\section{References}

[1] Fama E F, Fisher L, Jensen M C, et al. The adjustment of stock prices to new information. International Economic Review, 1969, 10: 1-21.

[2] Ikenberry D L, Rankine G, Stice E K. What do stock splits really signal? Journal of Financial and Quantitative Analysis, 1996, 31(3): 357-375.

[3] Desai H, Jain P C. Long-run common stock returns following stock splits and reverse splits. Journal of Business, 1997, 70: 409-433.

[4] Byun J, Rozeff M S. Long-run performance after stock splits: 1927 to 1996. Journal of Finance, 2003, LVIII(3): 1063-1085.

[5] Li F Y, Liu M H, Shi Y D. Institutional ownership around stock splits. Pacific-Basin Finance Journal, 2017, 46: $14-40$.

[6] So R W, Tse Y. Rationality of stock splits: The target-price habit hypothesis. Review of Quantitative Finance and Accounting, 2000, 14: 67-84.

[7] Payne J L, Thomas W B. The implications of using stock-split adjusted I/B/E/S data in empirical research. Accounting Review, 2003, 78(4): 1049-1067.

[8] Hardin III W G, Liano K, Huang G C. REIT stock splits and market efficiency. Journal of Real Estate Finance and Economics, 2005, 30(3): 297-315.

[9] Huang G C, Liano K, Pan M S. Do stock splits signal future profitability? Review of Quantitative Finance and Accounting, 2006, 26: 347-367.

[10] Chern K Y, Tandon K, Yu S, et al. The information content of stock split announcements: Do options matter? Journal of Banking \& Finance, 2008, 32: 930-946.

[11] Chen H H, Nguyen H H, Singal V. The information content of stock splits. Journal of Banking \& Finance, 2011, 35: 2454-2467.

[12] Huang G C, Liano K, Pan M S. REIT stock splits and liquidity changes. Journal of Real Estate Finance and Economics, 2011, 43: 527-547.

[13] Steve J, Stretcher R. News and noise: Do investors react to stock split announcements differently during periods of high and low market volatility? Journal of Economics and Finance, 2011, 35: 71-78.

[14] Huang G C, Liano K, Pan M S. The effects of stock splits on stock liquidity. Journal of Economics and Finance, 2015, 39: 119-135.

[15] Zeng Y, McLaren J. The impact of large public sales of Government assets: Empirical evidence from the Chinese stock markets on a gradual and offer-to-get approach. Review of Quantitative Finance and Accounting, 2015, 45: 137-173.

[16] Hu M, Chao C C, Malone C, et al. Real determinants of stock split announcements. International Review of Economics and Finance, 2017, 51: 574-598.

[17] Schultz P. Stock splits, tick size, and sponsorship. Journal of Finance, 2000, LV(1): 429-450.

[18] Nguyen V, Tran A, Zeckhauser R. Stock splits to profit insider trading: Lessons from an emerging market. Journal of International Money and Finance, 2017, 74: 69-87.

[19] Minnick K, Raman K. Why are stock splits declining? Financial Management, 2013, 43(1): 29-60. 
[20] Crutchley C E, Swidler S. Multiple reverse stock splits (investors beware!) Journal of Economics and Finance, 2015, 39: 357-369.

\section{Appendix}

\section{A.1 The proof of Theorem 2}

Proof Assume a stock of intrinsic value $X$ will be split by the strategy that a share is split into $m$ shares, where $m>1$. And assume its expectation value of intrinsic value will change from $E[X]$ to $E[X]+\Delta$ just at stock split, where $\Delta$ is a known number. Denote the stock price and the risk size of intrinsic value just before the stock split by $P_{0}$ and $\rho_{0}$ respectively, then its ex-right price and risk size are $\alpha P_{0}$ and $\alpha \rho_{0}$. And denote its corresponding reasonable price just after ex-right by $P_{1}$ and $\alpha=\frac{1}{m}$, then $\alpha \in(0,1)$.

In a risk-aversive financial market, for a rational investor we can regard the stock price just before the stock split equals its reasonable price. It yields from (1) that

$$
P_{0}+\eta\left(\rho_{0}\right)=E[X]
$$

and

$$
P_{1}+\eta\left(\alpha \rho_{0}\right)=\alpha(E[X]-\Delta) .
$$

On the other hand, it follows from (6) that there exists $C_{0}$ such that

$$
\alpha P_{0}+\eta\left(\alpha \rho_{0}\right)=C_{0}
$$

Case 1. Obviously, $\alpha \eta\left(\rho_{0}\right)-\eta\left(\alpha \rho_{0}\right)<\alpha \Delta$ is equivalent to

$$
\alpha\left(P_{0}+\eta\left(\rho_{0}\right)-\Delta\right)<\alpha P_{0}+\eta\left(\alpha \rho_{0}\right) .
$$

It follows from (6) and (9) that

$$
\alpha(E[X]-\Delta)<\alpha P_{0}+\eta\left(\alpha \rho_{0}\right) .
$$

It equivalently yields from (7) and (10) that

$$
P_{1}<\alpha P_{0},
$$

which means stock price should go down for a stock split.

Case 2. Obviously, $\alpha \eta\left(\rho_{0}\right)-\eta\left(\alpha \rho_{0}\right)>\alpha \Delta$ is equivalent to

$$
\alpha\left(P_{0}+\eta\left(\rho_{0}\right)-\Delta\right)>\alpha P_{0}+\eta\left(\alpha \rho_{0}\right) .
$$

It follows from (6) and (12) that

$$
\alpha(E[X]-\Delta)>\alpha P_{0}+\eta\left(\alpha \rho_{0}\right) .
$$

It equivalently yields from (7) and (13) that

$$
P_{1}>\alpha P_{0},
$$


which means stock price should go up for a stock split.

Case 3. Obviously, $\alpha \eta\left(\rho_{0}\right)-\eta\left(\alpha \rho_{0}\right)=\alpha \Delta$ is equivalent to

$$
\alpha\left(P_{0}+\eta\left(\rho_{0}\right)-\Delta\right)=\alpha P_{0}+\eta\left(\alpha \rho_{0}\right) .
$$

It follows from (6) and (15) that

$$
\alpha(E[X]-\Delta)=\alpha P_{0}+\eta\left(\alpha \rho_{0}\right) .
$$

It equivalently yields from (7) and (16) that

$$
P_{1}=\alpha P_{0},
$$

which means a stock split doesn't make stock price changed.

\section{A.2 The proof of Theorem 3}

Proof Assume a stock of intrinsic value $X$ will be split by the strategy that a share is split into $m$ shares, where $m>1$. And assume its risk size of intrinsic value will change from $\rho_{0}$ to $\rho_{0}+\delta$ just at stock split, where $\delta$ is a known number. Denote the stock price just before the stock split by $P_{0}$, then its ex-right price and risk size are $\alpha P_{0}$ and $\alpha\left(\rho_{0}+\delta\right)$, where $\alpha=\frac{1}{m}$ and $\alpha \in(0,1)$. And denote its corresponding reasonable price just after ex-right by $P_{1}$.

In a risk-aversive financial market, for a rational investor we can regard the stock price just before the stock split equals its reasonable price. It yields from (1) that

$$
P_{0}+\eta\left(\rho_{0}\right)=E[X]
$$

and

$$
P_{1}+\eta\left(\alpha\left(\rho_{0}+\delta\right)\right)=\alpha E[X] .
$$

Case 1. Obviously, $\eta\left(\alpha\left(\rho_{0}+\delta\right)\right)>\alpha \eta\left(\rho_{0}\right)$ is equivalent to

$$
\alpha P_{0}+\eta\left(\alpha\left(\rho_{0}+\delta\right)\right)>\alpha\left(P_{0}+\eta\left(\rho_{0}\right)\right) .
$$

It follows from (18) and (20) that

$$
\alpha P_{0}+\eta\left(\alpha\left(\rho_{0}+\delta\right)\right)>\alpha E[X] .
$$

It equivalently yields from (19) and (21) that

$$
P_{1}<\alpha P_{0},
$$

which means stock price should go down for a stock split.

Case 2. Obviously, $\eta\left(\alpha\left(\rho_{0}+\delta\right)\right)<\alpha \eta\left(\rho_{0}\right)$ is equivalent to

$$
\alpha P_{0}+\eta\left(\alpha\left(\rho_{0}+\delta\right)\right)<\alpha\left(P_{0}+\eta\left(\rho_{0}\right)\right) .
$$

It follows from (18) and (23) that

$$
\alpha P_{0}+\eta\left(\alpha\left(\rho_{0}+\delta\right)\right)<\alpha E[X] .
$$


It equivalently yields from (19) and (24) that

$$
P_{1}>\alpha P_{0},
$$

which means stock price should go up for a stock split.

Case 3. Obviously, $\eta\left(\alpha\left(\rho_{0}+\delta\right)\right)=\alpha \eta\left(\rho_{0}\right)$ is equivalent to

$$
\alpha P_{0}+\eta\left(\alpha\left(\rho_{0}+\delta\right)\right)=\alpha\left(P_{0}+\eta\left(\rho_{0}\right)\right) .
$$

It follows from (18) and (26) that

$$
\alpha P_{0}+\eta\left(\alpha\left(\rho_{0}+\delta\right)\right)=\alpha E[X] .
$$

It equivalently yields from (19) and (27) that

$$
P_{1}=\alpha P_{0},
$$

which means a stock split doesn't make stock price changed. 